\author{
United Nations Educational Scientific and Cultural Organization \\ and \\ International Atomic Energy Agency \\ THE ABDUS SALAM INTERNATIONAL CENTRE FOR THEORETICAL PHYSICS
}

\title{
SHEAR VISCOSITY OF LIQUID MIXTURES: MASS DEPENDENCE
}

\author{
Rohan Kaushal \\ Department of Physics, Panjab University, Chandigarh-160014, India \\ and \\ K. Tankeshwar ${ }^{1}$ \\ Department of Physics, Panjab University, Chandigarh-160014, India \\ and \\ The Abdus Salam International Centre for Theoretical Physics, Trieste, Italy.
}

\begin{abstract}
Expressions for zeroth, second, and fourth sum rules of transverse stress autocorrelation function of two component fluid have been derived. These sum rules and Mori's memory function formalism have been used to study shear viscosity of Ar-Kr and isotopic mixtures. It has been found that theoretical result is in good agreement with the computer simulation result for the Ar-Kr mixture. The mass dependence of shear viscosity for different mole fraction shows that deviation from ideal linear model comes even from mass difference in two species of fluid mixture. At higher mass ratio shear viscosity of mixture is not explained by any of the emperical model.
\end{abstract}

MIRAMARE - TRIESTE

June 2002

\footnotetext{
${ }^{1}$ Regular Associate of the Abdus Salam ICTP.
} 


\section{Introduction}

An appreciable progress has been made for studying diffusion, viscosity and thermal conductivity of one component fluids. This has become possible due to the collective efforts of experiments, theoretical studies, and computer simulations. The transport properties of the two component fluids have also been of interest among physicists and chemists due to their composition dependence and more recently from the point of view of glass transition. Extensive computer simulation studies have been carried out to investigate transport phenomena in Lennard Jones fluid[1-3], soft sphere fluid [4], and hard sphere fluid [5] mixtures. The composition dependence of diffusion and shear viscosity has been one of the aim of these studies. The composition dependence of shear viscosity of binary mixture from a theoretical point of view is not yet fully understood. There exist models like ideal linear model [6] and an exponential model [7] for composition dependence. But it has been found that there are deviations [3] from the predictions of these models. Some microscopic theoretical studies based on mode coupling approach [8] and kinetic theory approach [9] have also been made. Recently, Mukherjee et al [10] have studied the composition dependence of shear viscosity of modeled binary mixture system. In this modeled system, the two species have the same mass and same size but different interaction strength. It is found that deviations from ideality are enormous in such a system. In real system two species differ in mass, size, and interaction strength simultaneously. In the present work, we study equimolar Ar-Kr mixture and a composition dependence of shear viscosity of modeled mixture in which the two species are allowed to have only different masses. Similar studies have been carried out earlier $[11,12]$ for self-diffusion coefficients predicting weak mass dependence of self diffusion in agreement with simulation studies $[13,14]$.

To study the shear viscosity of mixture we have used the time correlation function approach coupled with the Mori -Zwanzig memory function formalism. In this approach, memory function is the basic quantity to be determined. The memory function can be calculated microscopically using binary collision [15] and mode coupling theories [16]. Though, there exists microscopic expression [17] for binary contribution but only for one component system. Alternatively, one can study viscosity coefficient by investigating short time properties of transverse stress auto correlation (TSAC) function and modeled memory function. In the present work we have derived zeroth, second, and fourth sum rules of TSAC function. These expressions are quite general, applicable to any two component system, and involve partial pair and triplet correlation functions. These sum rules have been computed numerically for equimolar Ar-Kr mixture. The result obtained for shear viscosity is in good agreement with computer simulation result[2].

In order to investigate the effect of mass on shear viscosity of mixture, we have studied its composition dependence for various mass ratios of two species. It has been found that even the mass difference in two species leads to deviations from emperical linear and exponential models.

The layout of the paper is as follows: In the second section, we present theoretical formalism 
and derivation of expressions for the sum rules of TSAC function for a general two component system and isotopic system. In the third section, results and discussion are given. The fourth section consists of a brief summary and conclusion.

\section{Theoretical Formalism}

The Green-Kubo expression relating transverse stress auto-correlation function, $S(t)$ to viscosity is given by

$$
\eta=\frac{1}{k_{B} T V} \int_{0}^{\infty} S(t) d t
$$

where $k_{B}, \mathrm{~T}$, and $\mathrm{V}$ are the Boltzmann's constant, absolute temperature and volume of the system, respectively. Here, $\mathrm{S}(\mathrm{t})$ is defined as

$$
S(t)=\left\langle J_{x y}(t) J_{x y}(0)\right\rangle
$$

with

$$
J_{x y}(t)=\sum_{i}^{N}\left[m_{i} v_{i y}(t) v_{i x}(t)+x_{i}(t) F_{i y}(t)\right] .
$$

In above equation $N=N_{1}+N_{2}$ is total number of particles labelled as 1 and 2. $x_{i}(t)$ and $v_{i x}(t)$ represent $x$ component of position and velocity of the $i^{t h}$ particle at time $t$, respectively. $v_{i y}(t)$ and $F_{i y}(t)$ are, respectively, $y$ components of velocity and force at time $t$. The angular brackets in Eq.(2) represent ensemble average. The TSAC function $S(t)$ involves interactions among like particles and unlike particles.

It is not yet possible to calculate $\mathrm{S}(\mathrm{t})$ exactly for a system of particles involving interactions. Therefore, we study the time evolution of the TSAC function by examining its short time properties. The short time expansion of $S(t)$ is given as

$$
S(t)=S_{0}-S_{2} \frac{t^{2}}{2 !}+S_{4} \frac{t^{4}}{4 !}+\ldots .
$$

where $S_{0}, S_{2}$, and $S_{4}$ are called zeroth, second, and fourth sum rules of the stress auto-correlation function, respectively.

\subsection{Expressions for Sum Rules}

\subsubsection{Two Component System}

The zeroth sum rule of TSAC function is defined as

$$
S_{0}=\left\langle J_{x y}(0) J_{x y}(0)\right\rangle
$$

After evaluating ensemble average in above equation, the expression obtained for $S_{0}$ is given as

$$
S_{0}=N\left(k_{B} T\right)^{2}+\frac{k_{B} T}{2} \sum_{\mu, \nu=1}^{2} n_{\mu} N_{\nu} \int d \mathbf{r} g^{\mu \nu}(r) x^{2} U_{y y}^{\mu \nu}
$$


Here, $n_{\mu}$ denotes the number density of species labelled as $\mu . g^{\mu \nu}(r)$ is pair correlation function between particles of species $\mu$ and $\nu$. In Eq.(6) and what follows, we use notation

$$
U_{\alpha_{1}, \alpha_{2}, \ldots \alpha_{n}}^{\mu \nu}(r)=\frac{\partial^{n} U^{\mu \nu}(r)}{\partial r_{\alpha_{1}}, \partial r_{\alpha_{2}}, \ldots, \partial r_{\alpha_{n}}}
$$

$U^{\mu \nu}(r)$, in the above expression is pair potential between the particles of species $\mu$ and $\nu . r_{\alpha_{n}}$ is $\alpha_{n}^{\text {th }}$ component of $\mathbf{r}$. Clearly expression for $S_{0}$ contains interaction among like species and unlike species of the two component system.

The derivation of the second sum rule of TSAC function for the two component fluids requires the calculation of ensemble average of product of the time derivative of dynamical variable $J_{x y}(t)$ at $t=0$ with itself. It is defined as

$$
S_{2}=\left\langle\dot{J}_{x y}(0) \dot{J}_{x y}(0)\right\rangle
$$

where $\dot{J}_{x y}$ represents first time derivative of the dynamical variable $J_{x y}(t)$. The expression for second sum rule, $S_{2}$ of the TSAC function for a two component system is obtained to be

$$
\begin{aligned}
S_{2}= & \left(k_{B} T\right)^{2} \sum_{\mu, \nu=1}^{2} \frac{n_{\mu} N_{\nu}}{m_{\mu}} \int d \mathbf{r} g^{\mu \nu}(r)\left[7 U_{y y}^{\mu \nu}+6 x U_{y y x}^{\mu \nu}+\left(k_{B} T\right)^{-1} x^{2}\left(U_{y \alpha}^{\mu \nu}\right)^{2}\right] \\
& +\left(k_{B} T\right) \sum_{\mu, \nu=1}^{2} \frac{N_{\mu}}{m_{\mu}} n_{\mu} n_{\nu} \iint d \mathbf{r} d \mathbf{r}_{1} g_{3}^{\mu \nu}\left(\mathbf{r}, \mathbf{r}_{1}\right) x x_{1} U_{y \gamma}^{\mu \nu} U_{1 y \gamma}^{\mu \nu}
\end{aligned}
$$

where $g_{3}\left(\mathbf{r}, \mathbf{r}_{\mathbf{1}}\right)$ is triplet distribution function.

The fourth sum rule is defined as

$$
S_{4}=\left\langle\ddot{J}_{x y}(0) \ddot{J}_{x y}(0)\right\rangle \text {. }
$$

The expression for $S_{4}$, is expected to involve partial triplet and quadruplet distribution function in addition to pair correlation function. In the absence of knowledge of higher order partial correlation functions, we have restricted our calculations only upto two body terms. Moreover, in case of one component system it was noted [18] that three and four body contributions to the fourth sum rule are opposite in sign. The expression for $S_{4}$ involving only two body contributions thus obtained is given as

$$
\begin{aligned}
S_{4}= & \frac{\left(k_{B} T\right)^{2}}{2} \sum_{\mu, \nu=1}^{2} n_{\mu} N_{\nu}\left(\frac{1}{m_{\mu}}+\frac{1}{m_{\nu}}\right)^{2} \int d \mathbf{r} g^{\mu \nu}(r)\left[9\left(k_{B} T\right) U_{x x y y}^{\mu \nu}+16\left(U_{y \alpha}^{\mu \nu}\right)^{2}\right. \\
& +20\left(U_{x y}^{\mu \nu}\right)^{2}+15 U_{x x}^{\mu \nu} U_{y y}^{\mu \nu}+10 x U_{y y \alpha}^{\mu \nu} U_{x \alpha}^{\mu \nu}+26 x U_{x y \alpha}^{\mu \nu} U_{y \alpha}^{\mu \nu}+3 x^{2}\left(U_{y \alpha \beta}^{\mu \nu}\right)^{2} \\
& \left.+\left(k_{B} T\right)^{-1} x^{2} U_{y \alpha}^{\mu \nu} U_{y \beta}^{\mu \nu} U_{\alpha \beta}^{\mu \nu}\right] .
\end{aligned}
$$

From expressions for $S_{0}, S_{2}$, and $S_{4}$ given by Eqs.(6), (9) and (11), already known expressions[18] for one component system can be obtained when the number of particles of either species is put equal to zero. 


\subsubsection{Isotopic System}

We consider here a system in which two species differ only in their masses. The study of such a system is important to know the effect of mass and concentration on shear viscosity. Analytical expressions for the mass and concentration dependence of sum rules of the TSAC function for such an isotopic system are derived by allowing all particles to interact with same pair potential. Expression for zeroth sum rule is given by

$$
S_{0}\left(m_{2}\right)=S_{0}\left(m_{1}\right)
$$

This implies that $S_{0}$ does not depend on mass of particles. The mass and concentration dependence of $S_{2}\left(m_{1}, m_{2}\right)$, and $S_{4}\left(m_{1}, m_{2}\right)$, is respectively, given as

$$
S_{2}\left(m_{1}, m_{2}\right)=\left[c+\frac{(1-c) m_{1}}{m_{2}}\right] S_{2}\left(m_{1}\right)
$$

and

$$
S_{4}\left(m_{1}, m_{2}\right)=\frac{1}{2}\left[c(1+c)+(1-c)(2-c) \frac{m_{1}^{2}}{m_{2}^{2}}+2 c(1-c) \frac{m_{1}}{m_{2}}\right] S_{4}\left(m_{1}\right),
$$

where $c=\frac{N_{1}}{N}$ is concentration of particles having mass $m_{1} . S_{0}\left(m_{1}\right), S_{2}\left(m_{1}\right)$, and $S_{4}\left(m_{1}\right)$ are, respectively, given as

$$
\begin{aligned}
& S_{0}\left(m_{1}\right)=N\left(k_{B} T\right)^{2}+\frac{k_{B} T}{2} N n \int d \mathbf{r} g^{\mu \nu}(r) x^{2} U_{y y}^{\mu \nu} \\
S_{2}\left(m_{1}\right)= & \frac{N n\left(k_{B} T\right)^{2}}{m_{1}} \int d \mathbf{r} g(r)\left[7 U_{y y}+6 x U_{y y x}+\left(k_{B} T\right)^{-1} x^{2}\left(U_{y \alpha}\right)^{2}\right] \\
& +N k_{B} T n^{2} \iint d \mathbf{r} d \mathbf{r}_{1} g_{3}\left(\mathbf{r}, \mathbf{r}_{\mathbf{1}}\right) x x_{1} U_{y \gamma} U_{1 y \gamma}
\end{aligned}
$$

and

$$
\begin{aligned}
S_{4}\left(m_{1}\right)= & \frac{2 N n\left(k_{B} T\right)^{2}}{m_{1}^{2}} \int d \mathbf{r} g(r)\left[9\left(k_{B} T\right) U_{x x y y}+16\left(U_{y \alpha}\right)^{2}+20\left(U_{x \alpha}\right)^{2}+15 U_{x x} U_{y y}\right. \\
& +10 x U_{y y \alpha} U_{x \alpha}+26 x U_{x y \alpha} U_{y \alpha}+3 x^{2}\left(U_{y \alpha \beta}\right)^{2} \\
& \left.+\left(k_{B} T\right)^{-1} x^{2} U_{y \alpha} U_{y \beta} U_{\alpha \beta}\right] .
\end{aligned}
$$

The above expressions are zeorth, second, and fourth (two body only) sum rules of TSAC function of one component system.

\subsection{Expression for Shear Viscosity}

To calculate shear viscosity from the expression given by Eq.(1), one requires to know time evolution of TSAC function, $S(t)$. The exact evaluation of time evolution is possible only for a simplified description of atomic motion. Mori has shown, however, that time correlation 
functions obey $[19,20]$ an equation of motion which determines their time evolution and is given by

$$
\frac{d S(t)}{d t}=-\int_{0}^{t} M_{1}(t-\tau) S(\tau) d \tau
$$

where $M_{1}(t)$ is first order memory function defined as

$$
M_{1}(t)=\left\langle f_{1}(t) f_{1}^{*}(0)\right\rangle /\left\langle\left|f_{1}(0)\right|^{2}\right\rangle
$$

with

$$
f_{1}(t)=\exp \left(i Q_{1} \tilde{L} Q_{1} t\right) Q_{1} J
$$

Here, $Q_{1}$ is the operator projecting into the subspace orthogonal to the variable $J(t)$ and $\tilde{L}$ is a Liouville operator. The quantity most required, for the calculation of time evolution of the TSAC function from Mori's equation, is the memory function, $M_{1}(t)$. Though, there exist ways to calculate $M_{1}(t)$ in binary collision approximation[17] and with mode coupling theory. But, the binary part calculations are still not known for the two component system interacting via continuous interaction potential. On the other hand, several phenomenological forms $[21,22]$ for the calculation of $M_{1}(t)$ have been proposed. Following earlier work which has provided [18,22] reasonably good estimates of shear viscosity for one component fluid and the fact that Gaussian memory dominates at higher mass ratio[11], we choose

$$
M_{1}(t)=\operatorname{aexp}\left(-b t^{2} / 2\right)
$$

where, $a=M_{1}(t=0)=\frac{S_{2}}{S_{0}}$ and $b^{2}=\frac{S_{4}}{S_{2}}-\frac{S_{2}}{S_{0}}$, so that $S(t)$ satisfies sum rules upto fourth order.

Defining Fourier-Laplace transform as,

$$
\tilde{S}(\omega)=i \int_{0}^{\infty} \exp (i \omega t) S(t) d t
$$

one can obtain a relation for the time dependence of $\mathrm{S}(\mathrm{t})$ as,

$$
S(t)=\frac{1}{\pi} \int_{0}^{\infty} \cos (\omega t) S^{\prime \prime}(\omega) d t
$$

where $S^{\prime \prime}(\omega)$ is imaginary part of $\tilde{S}(\omega)$. Using Green-Kubo expression (Eq.(1)) a general expression for the viscosity can be obtained, which is given by

$$
\eta=-\frac{i}{V k_{B} T} \tilde{S}(\omega=0)
$$

From Eqs.(18),(21),(24), and writing $a$ and $b$ in terms of sum rules, we obtain an expression for the shear viscosity given as

$$
\eta=\sqrt{\left(\frac{2}{\pi}\right)}\left(\frac{n}{k_{B} T}\right)\left(\frac{S_{4}}{S_{2}}-\frac{S_{2}}{S_{0}}\right)^{\frac{1}{2}}\left(\frac{S_{0}^{2}}{S_{2}}\right) .
$$

We will use this equation of shear viscosity and expressions of $S_{0}, S_{2}$, and $S_{4}$ to study the $\mathrm{Ar}-\mathrm{Kr}$ and isotopic mixtures. 
Table 1: Values of sum rules $S_{0}, S_{2}$, and $S_{4}$. The value of $S_{0}$ is in units of $10^{-43} \mathrm{~J}^{2}, S_{22}$ and $S_{23}$ are in units of $10^{-17} J^{2} s^{-2}$ and that of $S_{4}$ in units of $10^{10} J^{2} s^{-4}$.

\begin{tabular}{|c|c|c|c|}
\hline & Ar-Ar & Ar-Kr & Kr-Kr \\
\hline$S_{0}$ & 106.804 & 289.066 & 220.316 \\
$S_{22}$ & 70.948 & 195.546 & 140.615 \\
$S_{23}$ & -9.823 & -33.280 & -16.516 \\
$S_{4}$ & 32.166 & 63.492 & 31.201 \\
\hline
\end{tabular}

\section{Results and Discussion}

To calculate shear viscosity from the expression given by Eq.(25), one requires to compute the sum rules $S_{0}, S_{2}$, and $S_{4}$. The zeroth sum rule contains only two body terms whereas, the expression for $S_{2}$ contains two and three body terms. In the present work, we have considered only two body terms in the fourth sum rule. After carrying out the angular integrations by using an analytical method, the sum rules have been computed numerically. The angularly integrated expressions are given in the Appendix. The numerical computation of the sum rules requires interatomic potential, partial pair and triplet correlation functions as input. Presentely, calculations have been carried out for equimolar mixture of Argon and Krypton by using simulation data [3] for the partial pair correlation. The interaction potential for such a system is Lennard-Jones(LJ) potential given as

$$
U(r)=4 \epsilon\left[\left(\frac{\sigma}{r}\right)^{12}-\left(\frac{\sigma}{r}\right)^{6}\right] .
$$

In the above expression of potential, value of $\epsilon$ for Ar-Ar is taken as $\epsilon_{11}=120 K \times k_{B}$ whereas for $\mathrm{Kr}-\mathrm{Kr} \epsilon_{22}=167 K \times k_{B}$. The value of interatomic distance for Ar-Ar is $\sigma_{11}=3.405 \stackrel{0}{A}$ and that for $\mathrm{Kr}-\mathrm{Kr}$ is $\sigma_{22}=3.633 \stackrel{0}{A}$. For interactions among unlike particles we have used $\epsilon_{12}=\sqrt{\epsilon_{11} \epsilon_{22}}$ and $\sigma_{12}=\frac{\sigma_{11}+\sigma_{22}}{2}$. The number density and temperature of the system are respectively, taken as $1.79 \times 10^{22} \mathrm{~cm}^{-3}$ and $121.7 \mathrm{~K}$, corresponding to state where simulation studies have been made. Numerical integrations have been carried out by using the Gauss quadrature method. The triplet contribution to second sum rule has been evaluated using the method explained in the appendix and is represented by $S_{23}$. The values of $S_{0}, S_{22}, S_{23}$, and $S_{4}$ for Ar-Ar, Ar-Kr and Kr-Kr are given in Table 1. Here $S_{22}$ represents the two body contribution to second sum rule. Using the values of $S_{0}, S_{2}$, and $S_{4}$ given in the Table 1 , we obtain $\eta=17.96 \times 10^{-7} \mathrm{Nsecm}^{-2}$ for equimolar Ar-Kr mixture. The corresponding computer simulation value [2] is $17.74 \times 10^{-7} \mathrm{NSecm}^{-2}$. Thus we see that there is very good agreement between ours and the computer simulation result.

The values of $S_{0}, S_{2}$ and $S_{4}$ for one component Ar system are $535.578 \times 10^{-43} \mathrm{~J}^{2}, 561.508 \times$ $10^{-17} J^{2} s^{-2}$ and $374.383 \times 10^{10} J^{2} s^{-4}$, respectively. For one component $\mathrm{Kr}$ system values of $S_{0}, S_{2}$ and $S_{4}$ are respectively, $1037.805 \times 10^{-43} J^{2}, 453.842 \times 10^{-17} J^{2} s^{-2}$ and $183.468 \times 10^{10} J^{2} s^{-4}$. Using these values in Eq.(25) we obtain viscosity for pure Ar, i.e., $\eta_{A r}=10.32 \times 10^{-7} \mathrm{NSecm}^{-2}$ 
and that of pure $\mathrm{Kr}$ as $\eta_{K r}=38.40 \times 10^{-7} \mathrm{Nsecm}^{-2}$. The corresponding computer simulation values are $\eta_{A r}=8.85 \times 10^{-7} \mathrm{NSecm}^{-2}$ and $\eta_{K r}=34.25 \times 10^{-7} \mathrm{Nsecm}^{-2}$. The shear viscosity of the Ar-Kr mixture as predicted by the linear relationship [6]

$$
\eta_{\text {mixture }}=c \eta_{K r}+(1-c) \eta_{A r}
$$

is $21.55 \times 10^{-7} \mathrm{Nsecm}^{-1}$, which is higher by about 20 percent than computer simulation or our theoretical result. The second relation [7] called exponential model determines shear viscosity of the mixture by

$$
\eta_{\text {mixture }}=\exp \left[c \ln \eta_{K r}+(1-c) \ln \eta_{A r}\right] .
$$

This predicts the value of shear viscosity of mixture to be $17.41 \times 10^{-7} \mathrm{Nsecm}^{-2}$. This value is more closer to the calculated/simulation value than the value predicted by Eq.(27). This finding is in agreement with the earlier investigation [2].

\subsection{Mass and Concentration Dependence}

In order to check the validity of the above linear and exponential models, we will study here a system where interactions among particles and size of the particles have been kept the same. The sum rules for $S_{2}$ and $S_{4}$ given by Eqs.(13) and (14) have been computed numerically for the various mass ratios and for different concentrations, whereas, $S_{0}$ is independent of the variations in mass and concentration. We have considered pure Ar system for the calculations for which values of sum rules are already given. These numerically calculated sum rules have been used to study the variation of viscosity with mass and concentration for the isotopic fluid. Fig.1 shows the variation of viscosity with mass ratio at different concenteration $\left(c=N_{1} / N\right.$ ) of particle of mass $m_{1}$ for an isotopic fluid. Knowing that shear viscosity of pure system varies as square root of mass, the mass and concentration dependence of shear viscosity for an isotopic fluid from the linear model is then given by

$$
\eta=\eta_{0}\left[c+(1-c) \sqrt{\frac{m_{2}}{m_{1}}}\right] .
$$

$\eta_{0}$ is the viscosity of one component system of particles with mass $m_{1}$ at $c=1$. The mass and concentration dependence of the shear viscosity for an isotopic fluid according to the exponential model is given as

$$
\eta=\eta_{0}\left[\sqrt{\frac{m_{2}}{m_{1}}}\right]^{(1-c)} .
$$

The results obtained from Eqs.(29) and (30) are also shown in Fig.1. It is seen from the figure that at $c=0.25$ linear model is able to explain the mass dependence but for $m_{2} / m_{1}<4$. At higher mass ratio the exponential model seems to be better. But at $c=0.5$ and $c=0.75$ the exponential model predicts better results than the linear model but only for small mass ratio. At higher mass ratio $(>4)$ there is significant departure from both models.

The composition dependence of the viscosity for small mass ratios $\left(m_{2} / m_{1}\right) 0.75,0.50$ and 0.25 is shown in Fig.2 along with the predictions of linear and exponential model. It can be seen 
from the figure that at mass ratio 0.75 both the models predict the concentration dependence in agreement with our theoretical results. However at $m_{2} / m_{1}=0.25$ the linear model has shown more deviation from our results than the exponential model. It is also noted that the larger the mass difference the larger is the deviation from predictions of emperical models. Thus it is an important finding that deviations from ideal linear model and the exponential model can be attributed to mass difference in two species. Here, it may be noted that the effect of interaction strength and atomic sizes, which is not included here, on the shear viscosity of mixtures may be equally or more significant.

To underline reasons for this deviation, we examine the behavior of $S_{4}$ and $S_{2}$ with change in concentration and mass ratio. It is noted that with an increase in $m_{1} / m_{2}, S_{2}$ increases linearly but $S_{4}$ increases as square of mass ratio as evident from Eqs.(13) and (14). It is also found that $S_{4}$, does not follow linear behavior with increase in concentration, whereas $S_{2}$ increases linearly. Thus it can be said that deviations from ideality comes from $S_{4}$ i.e., higher order sum rule.

\section{Summary and Conclusions}

We have derived expressions for the first three non-vanishing sum rules of the TSAC function and evaluated them numerically for a two component system. Using these expressions coupled with Mori's memory function formalism, we have calculated shear viscosity of equimolar Ar-Kr system. It is found that our approach provides an estimate of shear viscosity in agreement with computer simulation data. Further, it is found that the exponential model predicts better results than the linear model in agreement with earlier computer simulation results. In order to underline the reasons of deviation from the linear model we have studied mass and concentration dependence of isotopic mixture where the interactions among like and unlike particles are assumed to be the same. The study of the isotopic system reveals that at large mass ratio there is considerable deviation from the ideal linear model. Thus, one concludes that deviations from the linear model can be attributed to the mass difference in the two species of a mixture which in our case is coming from higher order sum rule. Further, for very large mass ratios there are deviations even from the exponential model. Thus, one finds that two emperical models can be useful only when two species of a mixture are not very different in their masses.

Acknowledgments: This work was done within the framework of the Associateship Scheme of the Abdus Salam International Centre for Theoretical Physics, Trieste, Italy. 


\section{Appendix:}

The angular integration of sum rules of the TSAC function is done using the method explained by Tankeshwar et al [18]. The angularly integrated expression for zeroth sum rule is given as

$$
S_{0}=N\left(k_{B} T\right)^{2}+\frac{2 \pi\left(k_{B} T\right)}{15} \sum_{\mu, \nu=1}^{2} n_{\mu} N_{\nu} \int_{0}^{\infty} d r g^{\mu \nu}(r) r^{4}\left[A_{\mu \nu} r^{2}+5 B_{\mu \nu}\right]
$$

where $B=\frac{1}{r} \frac{\partial U(r)}{\partial r} \quad$ and $A=\frac{1}{r} \frac{\partial B}{\partial r}$. The subscripts $\mu \nu$ on A and B imply that interaction is among $\mu$ and $\nu$ species of the sytem. The angularly integrated expression of two body contribution to second sum rules is given as

$$
\begin{gathered}
S_{22}=\left(k_{B} T\right)^{2} \sum_{\mu, \nu=1}^{2} \frac{4 \pi n_{\mu} N_{\nu}}{m_{\mu}} \int_{0}^{\infty} d r g^{\mu \nu}(r) r^{2}\left[7 B_{\mu \nu}+\frac{2}{5} C_{\mu \nu} r^{4}+\frac{13}{3} A_{\mu \nu} r^{2}\right. \\
\left.+\left(15 k_{B} T\right)^{-1} r^{2}\left(A_{\mu \nu}^{2} r^{4}+5 B_{\mu \nu}^{2}+2 A_{\mu \nu} B_{\mu \nu} r^{2}\right)\right]
\end{gathered}
$$

where, $C=\frac{1}{r} \frac{\partial A}{\partial r}$. The term containing the three body distribution function in $S_{2}$ is angularly integrated using an addition theorem which finally provides

$$
\begin{gathered}
S_{23}=\frac{8 \pi^{2} k_{B} T}{15} \sum_{\gamma=1}^{2} \frac{N_{\gamma}}{m_{\gamma}} \sum_{\mu, \nu=1}^{2} n_{\mu} n_{\nu} \int_{0}^{\infty} \int_{0}^{\infty} d r d r^{\prime} \int_{-1}^{+1} d \xi g_{3}^{\mu \nu}\left(\mathbf{r}, \mathbf{r}^{\prime}\right) \xi\left[10 B_{\mu \nu} B_{\mu \nu}^{\prime}\right. \\
\left.+2 A_{\mu \nu}^{\prime} B_{\mu \nu} r^{2}+2 A_{\mu \nu} B_{\mu \nu}^{\prime} r^{2}+A_{\mu \nu} A_{\mu \nu}^{\prime} r^{2} r^{2}\left(3 \xi^{2}-1\right)\right],
\end{gathered}
$$

where $\xi$ is cosine of angle between $\mathbf{r}$ and $\mathbf{r}^{\prime}$. Since, the information about partial $g_{3}(\mathbf{r}, \mathbf{r} /)$ is not available, we approximate it using a method as follows:

First we write $S_{23}$ in reduced units i.e., length in terms of $\sigma$, and energy in terms of $\epsilon$, so that the integral involved is dimensionless. The expression thus obtained is given as

$$
S_{23}=\frac{8 \pi^{2} k_{B} T}{15} \sum_{\gamma=1}^{2} \frac{N_{\gamma}}{m_{\gamma}} \sum_{\alpha, \beta=1}^{2} n_{\alpha} n_{\beta} \epsilon_{\alpha \beta}^{2} \sigma_{\alpha \beta}^{2} I_{\alpha \beta}
$$

where

$$
\begin{aligned}
& I_{\alpha \beta}=\int_{0}^{\infty} \int_{0}^{\infty} d r^{*} d r^{*} \int_{-1}^{+1} d \xi g_{3}^{\alpha \beta}\left(r, r^{\prime}\right) \xi\left[10 B_{\alpha \beta}^{*} B_{\alpha \beta}^{*}+2 A_{\alpha \beta}^{*} B_{\alpha \beta}^{*}\left(r^{\prime *}\right)^{2}\right. \\
& \left.\quad+2 A_{\alpha \beta}^{*} B_{\alpha \beta}^{*}\left(r^{*}\right)^{2}+A_{\alpha \beta}^{\prime *} A_{\alpha \beta}^{*}\left(r^{*}\right)^{2}\left(r^{\prime *}\right)^{2}\left(3 \xi^{2}-1\right)\right]
\end{aligned}
$$

The superscript $*$ means that the respective quantities are in reduced units. We approximate the integrand to have same value for all partial triplet correlation function, true in isotopic system, but with their weighted values in terms of their different strength of potential and atomic size. The integral $I_{\alpha \beta}$ has been calculated using superposition approximation.

The angular integration of the fourth sum rule of the TSAC function involving two body distribution function is given as 


$$
\begin{aligned}
& S_{4}=\frac{2 \pi\left(k_{B} T\right)^{2}}{15} \sum_{\mu, \nu=1}^{2} n_{\mu} N_{\nu}\left(\frac{1}{m_{\mu}}+\frac{1}{m_{\nu}}\right)^{2} \int_{0}^{\infty} d r g^{\mu \nu}(r) r^{2}\left[9 ( k _ { B } T ) \left(D_{\mu \nu} r^{4}+5 A_{\mu \nu}\right.\right. \\
& \left.\quad+10 C_{\mu \nu} r^{2}\right)+294 A_{\mu \nu}^{2} r^{4}+465 B_{\mu \nu}^{2}+490 A_{\mu \nu} B_{\mu \nu} r^{2}+54 A_{\mu \nu} C_{\mu \nu} r^{6}+36 B_{\mu \nu} \\
& \left.\times C_{\mu \nu} r^{4}+r^{2}\left(k_{B} T\right)^{-1}\left(A_{\mu \nu}^{3} r^{6}+3 B_{\mu \nu} A_{\mu \nu}^{2} r^{4}+3 A_{\mu \nu} B_{\mu \nu}^{2} r^{2}+5 B_{\mu \nu}^{3}\right)+3 C_{\mu \nu}^{2} r^{8}\right]
\end{aligned}
$$

where $D=\frac{1}{r} \frac{\partial C}{\partial r}$. Expressions given here are suitable for numerical integration.

\section{References}

[1] D. M. Heyes J. Chem.Phys, 96, 2217 (1992).

[2] S. H. Lee and P.T. Cummings, J. Chem. Phys. 99, 3919, (1993).

[3] Y. Zhou and G. H. Miller, Phys. Rev. E 53, 1587 (1996)

[4] D.J. Evans and H.J. M. Hanley, Phys.Rev.A 20,1648 (1979).

[5] J. J. Erpenbeck, Phys.Rev. 48, 223 (1993).

[6] G.W. Castellan, Physical Chemistry (Addison Wesley Reading 1971).

[7] R.C. Reid, J.M. Prausnitz, and T.K. Sherwood, The properties of Liquids and Gases 3rd Ed. (McGrawHill- New York, 1977).

[8] S. Sinha and M. Marchetti, Phys.Rev A 46, 4942 (1992).

[9] J.M. Kicad, S.Perz and E.G.D.Cohen, Phys. Rev.A 38, 3628 (1988).

[10] A. Mukherjee, G.Srinivas and B.Baghchi, Phys.Rev Lett. 86, 5926 (2001).

[11] K. Tankeshwar J.Phys.:Condens.Matter 7, 9715 (1995).

[12] R. Sharma, K. Tankeshwar and K.C. Sharma, Phys.Rev.E 59, 460 (1999).

[13] M. J. Nuevo, J.J.Morales and D.M. Heyes, Phys. Rev.B 51, 2026 (1995).

[14] M.J. Nuevo and J.J. Morales, Phys. Lett. A 178, 114 (1993).

[15] K.N. Pathak, S. Ranganathan and R.E. Johnson, Phys.Rev.E 50, 1135 (1994).

[16] J. Bosse, W. Götze and M. Lücke, Phys.Rev.A 17, 434 (1978).

[17] R.K. Sharma, K. Tankeshwar, K.N. Pathak, S. Ranganathan and R.E.Johnson, J.Chem.Phys.108, 2919 (1998). 
[18] K. Tankeshwar, K. N. Pathak and S. Ranganathan J.Phys.C: Solid State Phys. 21, 3607 (1988).

[19] J. P. Boon and S. Yip, Molecular Hydrodynamics (McGraw Hill ) (1980).

[20] J. P. Hansen and I. R. McDonald Theory of Simple Liquids (New York: Academic Press) (1986).

[21] P.C. Martin and S. Yip, Phys.Rev. 170, 151 (1968).

[22] K. Tankeshwar, K. N. Pathak and S. Ranganathan, J. Phys.: Condens.Matter 8, 10847 (1996). 
Figure 1: Variation of Shear viscosity of isotopic mixture with mass ratio of two species at $\mathrm{c}=0.25,0.5$ and 0.75 . Our results are represented by lines. Diamonds, squares and open triangles are the results from the linear model and,$+ \times$ and ${ }^{*}$ are from exponential models for $\mathrm{c}=0.25,0.5$ and 0.75 , respectively. 
Figure 2: Variation of Shear viscosity of isotopic mixture with concentration at different mass ratio $m_{2} / m_{1}=0.25,0.5$ and 0.75 . Our results are represented by lines. Diamonds, squares and open triangles are the results from the linear model and,$+ \times$ and $*$ are from exponential models for $m_{2} / m_{1}=0.25,0.5$ and 0.75 , respectively. 